\title{
DIE ERSTELLUNG EINES INTERAKTIVMEDIUMS ZUM LERNEN DER DEUTSCHEN GRAMMATIK
}

\author{
Ari Syahyati Dalimunte \\ Ahmad Bengar Harahap \\ Risnovita Sari
}

\begin{abstract}
AUSZUG
Das Ziel dieser Untersuchung ist es, die beweglichen animierten Texten mit dem Thema „, der Relativsatz “zu erstellen. Die Erstellung wird in dieser Untersuchung angewendet. Der Prozess der Erstellung eines Interaktivmediums „Swish Max” zum Lernen der deutschen Grammatik besteht aus der Erklärung der Phasen von ADDIE Theorie. Darunter sind: (1) Analyse, (2)Design/ Konzept, (3) Entwicklung, (4) Implementation und (5) Evaluation. Die Datenquelle dieser Untersuchung werden aus dem Internet von Webseiten und die Bücher genommen.Die Daten liefern animierte Texten, Tonen und Bildern. Das Ergebnis der Erstellung eines Interaktivmediums Swish Max zum Lernen der deutschen Grammatik ist45 Slides, 18.063 KB und 9.684 KB.Die Bewertung von der Experten über die Qualität des Ergebnissesder Erstellung eines Interaktivmediums zum Lernen der deutschen Grammatikist sehr gut.
\end{abstract}

\section{Schlüsswörter: Swish Max, Grammatik, Der Relativsatz.}

\section{EINLEITUNG}

Deutsch als Fremdsprache ist eine interessante Sprache, denn es gibt viele Unterschiede zu unserer Muttersprache. Eines der Ziele beim Deutsch lernen ist es, dass die Studenten mündlich und schriftlich kommunizieren können. In der Realität ist die Fähigkeit der Studenten aber nicht meist optimal. Während des Lernprozesses meinen die Studenten, dass Deutsch ein schwieriges Unterrichtsfach ist. Sie haben große Schwierigkeiten, die Hausaufgabe aus dem Deutschlernbuch zu erledigen. Es gibt aber auch andere Wege für die Deutschlernenden, um Deutsch zu lernen. Eine der Bemühungen, die die Lehrer tun können, um die Qualität der Bildung zu verbessern, ist die Verwendung unterschiedliches Lernmedium. Lernmedium können den Lernprozess unterstützen,interessanter gestalten und motivieren. Die Studenten verstehen die Aufgaben so leichter. Basierend auf Beobachtungen, benutzen die Lehrer und die Dozenten nur wenige Interaktivmedien. Deshalb denken die Studenten, dass die deutsche Sprache schwierig und wenig attraktiv ist und sie langweilen sich schneller im Unterricht. 
Heutzutage entwickelt sich die Informationstechnologie sehr schnell. Die verwendete Technologie ist immer Kompliziert, auch in der Bildung. Der Computer ist eines der Medien, die oft an Schulen und an der Universität verwendet werden. Lernmedium können Audio-Medien, visuelle Medien, audiovisuelle Medien, interaktive Medien, usw sein. Das Mediumdieser als Mittel, um Nachrichtenzu übermitteln. Das Interaktivmedium ist eines davon.

Die Interaktive Medium ist eine Medien-Nachrichten zwischen der Lehrern und der Schülern, die die Kommunikation zwischen Mensch und Technik durch Systeme und Software ist. (tania in Website 2015). Der Lernprozess kann überall und jederzeit stattfinden. Die Studenten müssen denoch aktiv lernen, wenn Sieein Lernmedium verwenden. Normalerweise lernen die Studenten in einem begrenzten Raum. Aber mit Interaktivmedium kann man auch in räumlicher Entfernung voneinander lernen. Heutzutage gibt es viele neue Applikationen oder Softwares, die als Lermedium verwendet werdenkonnen.

Swish Max ist eine Software zur Erstellung von animierten Flash wie Macromedia Flash MX. DieserSoftware ist einfach zu verstehen und kann Texte, Bilder, und Tone in kürzester Zeit erstellen. Die Ergebnisse dieser Software können auf eine Webseite hochgeladen werden. Obwohl diese Applikation ein Programm für die Präsentation ist, kann die Einrichtung aber auch dazu verwendet werden, um interaktive Medium zu erstellen.

Bassierend auf der Observation, und dem Interview finden viele Studenten, dass Relativsatz schwierig ist. Es gibt Häufige, um das Verb im Satz abzulegen. Mit dieser Entwicklung wird mit diesen Relativsatz leichter zu verstehen und kann das Lernen statische Atmosphäre verringern, um ein interessantes Lernen, effektiv, effizient und angenehm zu schaffen.

\section{THEORETISCHE GRUNDLAGE}

\section{Das Entwicklungsmodell}

In dieser Untersuchung wirddas ADDIE Modell benutzt. Asrar (2013:118) beschreiben, dass das ADDIE Model als Anleitung beim Aufbau der Vorrichtung erfüllt und Trainingsprogramme effektiv, dynamisch und unterstützt die Ausbildung. Es gibt fünf Phasen, um ein Interaktivmedium zum Lernen deutscher Grammatik zu 
entwickeln : 1) Die Analyse 2) Das Design/Konzeption 3) Die Development/Entwicklung 4) Die Umsetzung und 5) Die Evaluation.

\section{Der Begriff Des Interaktivmediums}

Nach Seels und Glasgow in Arsyad (2006: 36) erklären dass interaktive Medium ein Leiferungsmedium ist, das das Video durch den Computer den Zuschauern präsentiert. Die Zuschauer in diesem Fall sind die Schüler, können das Video nicht nur sehen und hören, sondern auch aktiv reagieren. Diese Reaktion bestimmt die Geschwindigkeit und die Reihenfolge der Präsentation.

Interaktive Medium haben audio-visuelle Elemente (einschließlich Animation). Das Medium wird mit aktiver entwickelt und beinhalten Benutzeraktion.Eigenschaften dieses Mediums ist, dass die Schüler nicht nur die Aufmerksamkeit auf die Medien oder Gegenstände bezahlen, sondern auch im Ausland für die Interaktion gebraucht. Es gibt drei Arten von Interaktion, nämlich die erste Interaktion zeigen die Studenten mit einem Programm wie leer interagieren . Die zweite Interaktion interagieren die Schüler mit Maschinen, wie Computer oder Simulation. Die dritte Interaktion, die Lehrer organisieren Studenten, die Interaktion nicht programmiert sind.

Aus der obigen Definition kann geschlossen werden, dass interaktive Medium ein Vermittler-Tool mit der Nutzung von Computern unter Verwendung von Elementen wie Voice (Audio), Bilder (visuell) und Text entworfen ist, eine Botschaft zu vermitteln.

\section{Der Software ,Swish Max”}

Swish Max ist ein Präsentationsanwendungsprogramm, das die Animationen erstellen hat (Khairina in Wordpress 2014). Eigentlich es auch die Software Erstellung Animation verwendet werden, aber Swish Max ist relativ einfach. Swish Max ist sehr einfach zu bedienen, um eine komplexe Animationen mit Text Variation in kurzer Zeit zu produzieren. Swish Max ist einerSoftware die Erstellung von animierten Flash wie Macromedia Flash MX. Diese Applikation ist einfach zu verstehen. Diese Software kann Animation Texte, Bilder, und Tone in kürzester Zeit hinzufügen. Die Ergebnisse dieser Software können in die Webseite importiert werden. 
Eine andere Sache im Swish Max kann die Arbeit zum SWF-Format exportiert werden, wie das format von Macromedia Flash. Flash Player muss auf dem PC installiert werden, da die Animation spielen. Obwohl dieses Anwendungsprogramm ist eigentlich ein Programm, um Präsentationen zu erstellen, aber die Fasilität kann für Lernprogramme verwendet werden.

\section{UNTERSUCHUNGSMETHODE}

Diese Untersuchung ist eine Erstellungsuntersuchung. Es wird ein Interaktivmedium erstellt. In dieser Untersuchung wird das Entwicklungsmodell von Reiser und Mollenda benutzt, das ADDIE Modell heißt.

\section{Die Daten und Die Datenquelle}

Die Daten dieser Untersuchung sind die Relativsätze im Lehr- und Übungsbuch der deutschen Grammatik und Studio DA2.

\section{Der Untersuchungsort}

Diese Untersuchung wird an der Deutschabteilung in der Fakultät für Sprachen und Kunst ander staatlichen Universität von Medan durchgeführt.

\section{Die Skizze der Untersuchung}

Diese Untersuchung benutzt die Theorie von Reiser und Mollenda. Diese wurdebereits in Kapitel II erklärt. Das sogenannte, ADDIE Modell,besteht aus fünf Phasen : (1) die Analyse (2) dem Design (3) der Entwicklung (4) derUmsetzung und (5) der Evaluation.

\section{ERGEBNIS DER UNTERSUCHUNG}

\section{Der Prozess der Erstellung}

\section{Analyse}

In der ersten Phase wird eine Leistungsanalyse durchgeführt. In dieser Phase wurde zunächst eine Umfrage unter 25 Studenten am 16. Mai 2017 an der Deutschabteilung durchgeführt. Aus den Antworten der Umfrage ergibt sich, dass die 
Studenten an der Deutschabteilung noch Schwierigkeiten beim Deutschlernen haben, z. B. beim der Relativsatz. Die Studenten langweilen sich. Die Studenten brauchen das neue Lernmedium, z.B das Interaktivmedium. Die Studenten fühlen sehr gluck, wenn das Lernen das Lernmedium verwendet wird.Nach der Umfrage wurde ein interessantes Medium gesucht, um ein ansprechendes Interaktivmedium zu entwickeln. Es gibt viele Arten von Medien, die im Unterricht verwendet werden können, sie sind : visuelles Medium, audio Medium, audio-visuelles Medium, und Interaktivmedium.

Hier wurde das Interaktivmedium gewählt. Das Interaktivmedium benuzt die Swish Max Applikation. Sie finden, dass Swish Max ein interessantes und ein neues Interaktivmedium ist. Bei dieser Untersuchung erstellte die Verfasserin ein Interaktivmedium mit Swish Max als Lernmedium zum Lernen der deutschen Grammatik. Die Verfasserin entwickelte das Thema „der Relativsatz” mit dem Interaktivmedium “Swish Max”. Mit Swish Max können animierte Texte, Bilder und Tönen erzeugt werden, so dass die Studenten einfacher „der Relativsatz” verstehen können.

\section{Design / Konzept}

Zweite Phase ist die Folge der ersten Phase. Nachdem in der ersten Phase Informationen über den Gebrauch des Interaktivmediums durch die Studenten gewonnen wurden, wird in dieser Phase das Konzept des Interaktivmediums mit der Swish Max Applikation entworfen, um ein interessantes Interaktivmedium zu erstellen.

\section{Development / Entwicklung}

In dieser Phase wird das Design aus der zweiten Phase verwirklicht. Nach Bildern und Tönen wurde bereits gesucht. In dieser Phase kann das Interaktivmedium auf Grundlage des gepanten Designs entwickelt werden. In dieser Phase wird der Gebrauch der Software Swish Max beschreiben.Bevor das Interaktivmedium mit der Swish Max Software entworfen wird, werden die Schritte vorgestellt, die bei der Gestaltung des Designs des Interaktivmediums berücksichtig werden müssen.

Um das Programm der Swish Max Software zu bedienen, drücken Sie Start, all programs, Swish Max, sodass das Welcome layout angezeigt wird. 


\section{Umsetzung / Implementation}

In dieser Phase wurde schon das Interaktivmedium mit der Swish MaxSoftware erstellt. Nun wird eine Validierung durchgeführt. So kann herausgefunden werden, ob das Interaktivmedium mit der Swish Max Software schon gut genug ist oder noch verbessert werden sollte. Nach der Validierung wird das Interaktivmedium mit der Swish Max Software verbessert. Dieses Interaktivmedium wird von einer expertin geprüft. Die Expertin ist eine Computer Dozentin, die viel über Medium wei $\beta$, Die Expertin hat die Daten schon korrigiert. Der Kommentar der Expertin ist sehr interessant.

Die Expertin hat das Interaktivmedium benotet : von 9 Kategorien der Aspekt der Evaluation der Evaluation gibt die Expertin die Note 4 für sieben Aspekt. Die Note 4 bedeutet sehr gut. Die Expertin die Note 3 für die übrigen Aspekte. Es gibt 2 Aspekte.

\section{Evaluation}

Das ist der letzte Schritt, um ein interessantes Interaktivmedium zu ertellen. Nach dem Kommentar, der Expertin werden Bilder Verändert. Die Praktikan hat auch kommentiert. Die Verwendung der Sprache ist noch nicht gut. Das ist zu schnell. Es gibt viele fehlen bei den Übungen. Die Verfasserin hat schon Interaktivmedium verbessert.

Basierend auf der obigen Meinung kann festgehalten werden, dass das Interaktivmedium mit seinen Bildern interessant gestaltet ist und besonders gut für das Thema „Der Relativsatz” ist. Allerdings ist die Erstellung des Interaktivmedium aufwendig, weil es schwer ist, den Player herunter zu laden.

\section{Das Ergebnis der Erstellung}

Das Design des Interaktivmedium ist vielfältig und kann auf unterschiedliche Weise entworfen werden. Die Haupseite (cover) des Interaktivediums ist „Die Grammatik „die Relativsätze”, auf der Hauptseite gibt es eine Titelseite und Informationen über die Verfasserin. Die Hauptseite sieht aus wie ein Einband, sie ist mit einem interessanten Scene gesteltet. Auf der zweiten Seiten gibt es die Menüliste. 
Es besteht 4 Menülisten namlich : 1) der Relativsatz, 2) die Einfügung, 3) die Verwendung, und 4) die Übungen. Die Menüliste ,der Relativsatz” enthalt Relativsatz, Relativpronomen und die Tabelle im Nominativ, Akkusativ, Dativ und Genitiv. Es gibt zwei Slides. Die Menüliste „die Einfügung” enthält die Beispiele zum Realtivsatz und die Teile des Relativsatzes (Haupsatz, Nebensatz, und Infinitivkonstruktionen). Es gibt 1 Slide und es gibt Ton und Bilder. Die Menüliste „Die Verwendung” enthält Beispiele für Relativsätze mit dem Relativpronomen im Nominativ, Akkusativ, Dativ und Genitiv. Es gibt 4 Beispiele und imgesamt 16 Beispiele. Die Beispiele benutzen Ton, Effect, und Bilder, die nächste Taste und die letzte Taste. Ein Slide hat ein Beispiel. Die Ende Menü „die Übungen” enthält Aufgaben für die Studenten. Es gibt 20 Aufgaben. Die Aufgaben sind Multiple Choice. Mit dem Interaktivmedium können Sie die Aufgaben hier beantwortet werden. Wenn die Antwort richtig ist, bekommen die Note 5. Die Gesamtnote ist 100. Es gibt 45 Slides. Es dauert 15 Minuten. Die Kapazität der beweglichen animierten Bilder, Texte, und Ton ist $18.063 \mathrm{~KB}$ file SWI und 9.684 KB file SWF. Dieses Interaktivmedium verwendet Bilder, Texte und Ton. Die Präsentationen mit Swish Max wird mit den umfassenden Erklärungen mit den Texten, Bildern, und Tonen mit dem Thema „Der Relativsatz” dargestellt. Mit diesen Elementen können interessante Interaktivmedium erstellt werden.

In der Zukunft kann diese Untersuchung als Interaktivmedium besonders um Deutschlehren und Deutschlernenden benutzt werden. Diese Erstellung des Interaktivmedium mit der Hilfe von Swish Max kann bei der Vermittlung des Relativsatzes helfen.Das Ergebnis für das Interaktivmedium, das mit der Swish Max Software entworfen wird, sieht folgendemaßen aus :

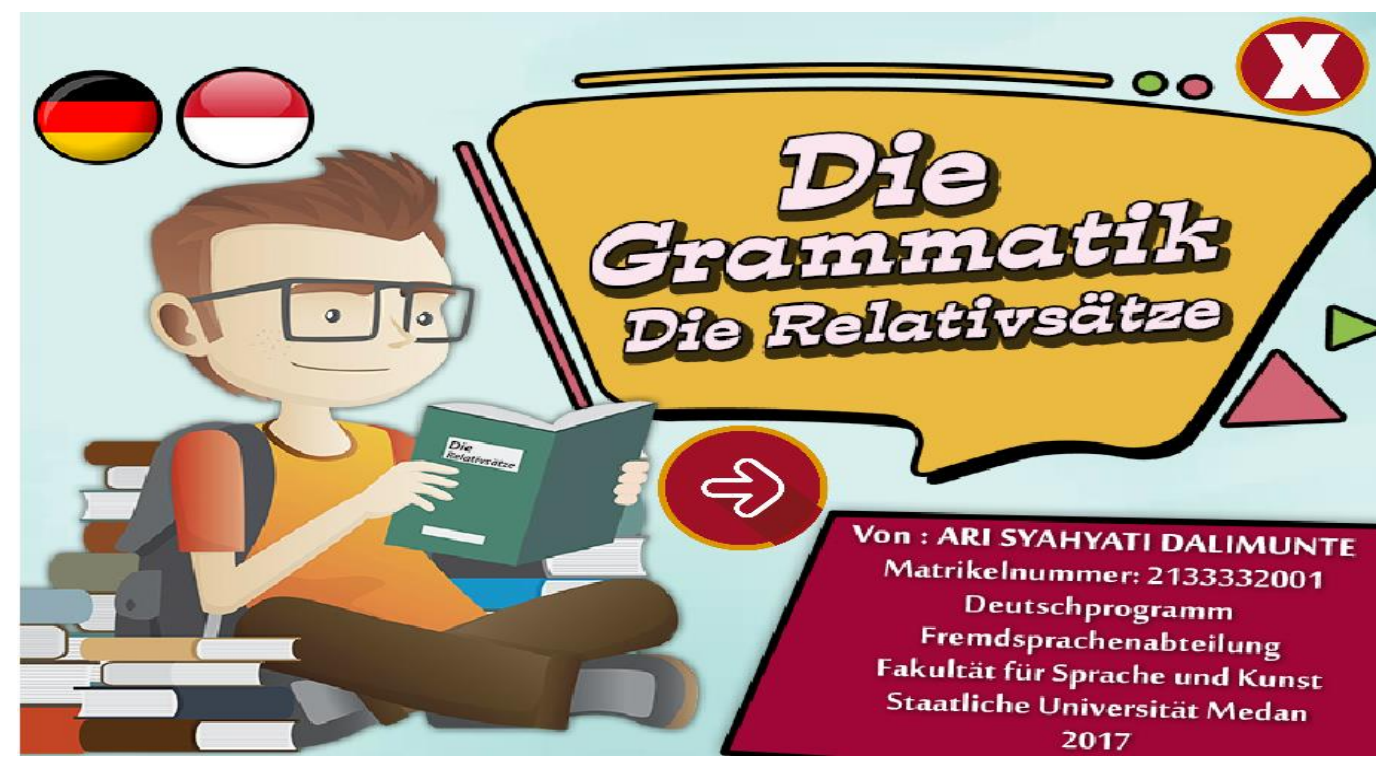


Bassierend auf den Ergebnissen der Erstellung des Interaktivmediums, das mit der Hilfe Swish Max Software zum Lernen der deutschen Grammatik „Der Relativsatz” wurde zusammengefasst. In dieser Untersuchung wird das ADDIE Modell Theorie benutzt. Diese Theorie besteht aus fünf Stufen, nämlich : (1) Analyse, (2) Design/Konzept, (3) Development/Entwicklung, (4) Implementation/Umsetzung, (5) Evaluation. In diesem Kapitel wird jede Stufe mit ihrem Ergebnis erklärt. In der Ermittlungsphase wird zuerst die Datemsammlung und die Problemidentifizierung durchgeführt. Aus den Antworten der Umfrage ergibt sich, dass die Studenten an der Deutschabteilung noch Schwierigkeiten beim Deutschlernen haben, z. B. beim der Relativsatz. Die Studenten brauchen die neues Medium. Sie finden, dass Swish Max interessantes Interaktivmedium und neues Interaktivmedium ist.

In dieser zweiten Phase wird das Konzept/Design des Interaktivmediums mit der Swish Max Software entworfen, um interessantes Interaktivmedium zu erstellen. Das Design wird an das Thema angepasst, das in der Swish Max Software integriert wird, um animierte Texte, Bilder, und Tonen zu erstellen. In dieser Phase wird der Relativsatz, die Einfügung, die Verwendung, und die Übungen erklärt.

Die nächste Phase ist Development/Entwicklungspahse. Das Lermaterial wird mithilfe der Swish MaxSoftware entwickelt. Das Interaktivmedium wurde schon mit Animierten Texten, Bildern, und Ton erstellt, so dass es interessantes Medium enthält. In dieser Phase gibt es viele Probleme. Z.B Wenn die Verfasserin die Tonen in die Datei kombinieren, muss die Tonen mit der Datei anpassen. So braucht die lange Stunden.

Die vierte Phase ist Implementation/Umsetzung. EineExpertin hat das Interaktivmedium mit dem Swish Max Software analysiert und korrigiert. Das Interaktivmedium bekommt die Note 3 über die Bilder und die Sprache. Die Verfasserin wird verbessert. Danach wird in der Implementation das erstellte Interaktivmedium mit dem Swih Max validiert. Die Expertin ist eine Computer Dozentin, die viel über Medium weiß. Die Expertin hat die Daten schon korrigiert. Der Kommentar der Expertin ist sehr interessant. Die Expertin hat das Interaktivmedium benotet : von 9 Kategorien der Aspekt der Evaluation. Der 
Evaluation gibt die Expertin die Note 4 für die sieben Aspekt. Die Note 4 bedeutet sehr gut. Es gibt 2 Aspekt Und die letzte Phase ist Evaluation. Die Expertin benotet das Interaktivmediums benotet 94,44 (sehr gut).

Bassierend auf der obigen Erklärung kann zusammengefasst werden, dass die Erstellung des Interaktivmediums mithilfe der Swish Max Software zum Thema „der Relativsatz” aus dem Buch Studio DA2 und im Lehr- und Übungsbuch der deutschen Grammatik sehr gut ist, sodass die Dozenten oder die Studenten das Interaktivmedium benutzen können.

\section{SCHLUSSFOLGERUNG}

Nach den Untersuchungsergebnissen fallen die Schlussfolgerungen folgendernmassen aus:

1. Der Prozes der Erstellung des Interaktivmediums mithilfe der Software "Swish Max” zum Lernen der deutschen Grammatik zum Thema „Relativsatz”. In diesem Prozess wurde die Phasen des ADDIE-Modells erklärt. Es gibt folgende Phasen: (1)Analyse (eine Umfrage machen und die Lehre analysieren), (2) Design/ Konzeption (Erklärungen über Relativsatz), Development/ Entwicklung (die Materialien mit der Swish Max Software entwickeln), (4) Implementation/ Umsetzung (die Expertin entscheidet, ob dieses Medium ist valid oder nicht), und (5) Evaluation (Abhängig von der Bewertung der Expertin wird das Interaktivmediums entweder verbessert oder nicht).

2. Das Ergebnis der Erstellung des Interaktivmediums mithilfe der Software "Swish Max" zum Lernen der deutschen Grammatik zum Thema „Relativsatz”.

a. In dieser Untersuchung wurden 43 Slides mit animierte um Text, Bild, Ton zu erstellt. 
b. Dieser Animierte Text benutzt Tonen.

c. Die Animierten Texte mit dem Thema „Relativsatz” wurden von einem Design experten evaluiert. Danach wird die Evaluation der animierten Texte korrigiert.

d. Das Interaktivmediums benotet : 9 Kategorien, die Note ist das Interaktivmediums 94.44 (sehr gut). Bassierend auf der Meinungen, dass das Interaktivmediums sehr interessant ist und das Ziel wird deutlich.

\section{VORSCHLÄGE}

Basierend auf den Ergebnissen und Schlussfolgerungen in dieser Untersuchun werden die folgenden Vorschläge gemacht:

1. Die Allgemeinen Leser können das Interaktivmediums mit Swish Max als Referenzquelle für weitere Untersuchungen verwenden und um Deutsch leichter zu Verstehen.

2. In der Zukunft können andere Erstellungsuntersuchungen im Deutschprogramm durchgeführt werden.

\section{LITERATURVERZEICHNIS}

Arif, Haryono dkk.2007.Teknik pembuatan presentasi menggunakan microsoft office power point dan swish max.Informatika:Bandung.

Arsyad, Azhar.1997.Media Pembelajaran.PT Raja Grafindo Persada:Jakarta

Hueber.2005.Lehr- und Übungsbuch der deutschen Grammatik.Deutschland.

Asrar Aspia dkk.2013.Media Pembelajaran.Perdana Publishing:Medan.

Rusman, dkk.2013.Pembelajaran Berbasis Teknologi Informasi dan Komunikasi. PT Raya Grafindo Persada:Jakarta.

Susilana, Rudi.2009.Media Pembelajaran.CV Wacana Prima:Bandung.

Sukiman. 2012. Pengembangan Media Pembelajaran. Yogyakarta: Pedajogja. 
Studia: Journal des Deutschsprogramms

p-ISSN 2301-6108

e-ISSN 2654-9573

https://jurnal.unimed.ac.id/2012/index.php/studia/index

Khairina.2014.(https://catatankhairina.wordpress.com/2014/12/23/swishmax-suatualternatif-media-pembelajaran-geometri-2/ hat gelesen den 24.April.2017 um 20.22 Uhr).

Yasin Nurdin.2015. Pengembangan Media Pembelajaran.

http://skripsinurdinyasin.blogspot.co.id/2015/08/pengembangan-mediapembelajaran.html gelesen den 27. April. 2017 um 19.21 Uhr.

Tania. 2015. Media Pembelajaran Interaktif.http://tania91.ilearning.me/laporankkp/bab-ii/2-8-media-pembelajaran-interaktif/ gesehen den 27. März. 2017 um 13.15 Uhr.

David. Program Aplikasi SwishMax. http://ahmaddavid10.blogspot.co.id/2014/10/vbehaviorurldefaultvmlo.html gelesen den 26. April. 2017 um 20.35 Uhr.

Ari Syahyati Dalimunte is an alumni of German language study program of Unimed, Medan 
Studia: Journal des Deutschsprogramms p-ISSN 2301-6108 e-ISSN 2654-9573

https://jurnal.unimed.ac.id/2012/index.php/studia/index 